\title{
Efficacy and safety of 1-week Helicobacter pylori eradication therapy and 7-week rebamipide treatment after endoscopic submucosal dissection of early gastric cancer in comparison with 8-week PPI standard treatment: a randomized, controlled, prospective, multicenter study
}

\author{
Kazuhide Higuchi $\cdot$ Toshihisa Takeuchi $\cdot$ Noriya Uedo $\cdot$ Yoji Takeuchi $\cdot$ Yuji Naito $\cdot$ \\ Nobuaki Yagi $\cdot$ Kazunari Tominaga $\cdot$ Hirohisa Machida $\cdot$ Takashi Tamada Yoshinori Morita \\ Shujiro Yazumi · Junichi Yamao • Mikitaka Iguchi · Takeshi Azuma
}

Received: 5 February 2014/ Accepted: 13 July 2014/Published online: 7 August 2014

(C) The International Gastric Cancer Association and The Japanese Gastric Cancer Association 2014

\begin{abstract}
Background Endoscopic submucosal dissection (ESD) has been developed for early gastric cancer (EGC). Helicobacter pylori eradication therapy has been reported to have a preventive effect against metachronous recurrence of EGC after ESD. However, the efficacy and safety of eradication therapy on ESD-induced ulcer healing are not clear. In a randomized control study, we compared the standard therapy (8-week proton pump inhibitor) and eradication therapy combined with subsequent treatment with 7-week rebamipide for healing ESD-induced ulcers. Methods A multicenter, randomized, open-label study was conducted. In group A, patients received $20 \mathrm{mg}$ of
\end{abstract}

\section{K. Higuchi $(\bowtie) \cdot T$. Takeuchi}

2nd Department of Internal Medicine, Osaka Medical College,

2-7 Daigaku-machi, Osaka, Takatsuki 569-8686, Japan

e-mail: higuchi@poh.osaka-med.ac.jp

N. Uedo $\cdot$ Y. Takeuchi

Department of Gastrointestinal Oncology, Osaka Medical Center for Cancer and Cardiovascular Diseases, Osaka, Japan

Y. Naito · N. Yagi

Department of Molecular Gastroenterology and Hepatology,

Graduate School of Medical Science, Kyoto Prefectural

University of Medicine, Kyoto, Japan

K. Tominaga $\cdot \mathrm{H}$. Machida

Department of Gastroenterology, Osaka City University

Graduate School of Medicine, Osaka, Japan

H. Machida

Machida Gastroenterical Hospital, Osaka, Japan

T. Tamada

Department of Gastroenterology, Takatsuki Red Cross Hospital,

Osaka, Takatsuki, Japan omeprazole for 56 days. In group $\mathrm{B}$, patients received $40 \mathrm{mg}$ of omeprazole, $1,500 \mathrm{mg}$ of amoxicillin, and $800 \mathrm{mg}$ of clarithromycin for 7 days, and then $300 \mathrm{mg}$ of rebamipide for 49 days. The primary end point was to evaluate the scarring ratio.

Results The scarring rate in group A was significantly higher than that in group B [85.0 \% (34/40) vs. $56.8 \%$ (21/ $37), P=0.011]$. The scarring rate of ulcers with an area $\geq 565.5 \mathrm{~mm}^{2}$ in group A was significantly higher than that in group B [78.9 \% (15/19) vs. $37.5 \%(6 / 16), P=0.018]$. There was no significant difference between the groups in the scarring rate of smaller ulcers. No serious adverse events were observed in any of the patients in either group.

\section{Y. Morita $\cdot$ T. Azuma}

Department of Internal Medicine, Division of Gastroenterology,

Kobe University Graduate School of Medicine, Hyogo, Kobe,

Japan

\section{S. Yazumi}

Digestive Disease Center, The Tazuke Kofukai Medical

Research Institute, Kitano Hospital, Osaka, Japan

J. Yamao

Third Department of Internal Medicine, Nara Medical

University, Nara, Japan

M. Iguchi

Second Department of Internal Medicine, Wakayama Medical

University, Wakayama, Japan 
Conclusion H. pylori eradication therapy and 7-week rebamipide monotherapy were not superior to PPI monotherapy, but this combination therapy for smaller sized ulcers was an optimal therapeutic option for healing. Serious adverse events were not observed in either group.

Keywords H. pylori eradication therapy - ESD .

Rebamipide · PPI · Artificial ulcer

\section{Introduction}

Endoscopic mucosal resection (EMR) has been established as a minimally invasive treatment for early stage gastric cancer (EGC) [1, 2]. However, en-bloc resection in patients with large tumors is often not indicated by EMR. Recently, endoscopic submucosal dissection (ESD) has been developed for EGC, and this procedure enables larger lesions to be resected, thereby yielding improved rates of successful en-bloc resection [3]. Uemura et al. demonstrated that Helicobacter pylori (H. pylori) had an important role in gastric carcinogenesis, since almost all non-cardiac gastric cancers develop from a background of $H$. pylori-infected mucosa [4]. In addition, $H$. pylori eradication therapy was effective for the prevention of metachronous recurrence of gastric cancer after ESD [5].

For artificial gastric ulcers after ESD, 8-week treatment with a proton pump inhibitor (PPI) or histamine 2 receptor antagonist was sufficient for healing [6,7]. On the other hand, eradication monotherapy is an alternative treatment for healing EMR-induced gastric ulcers [8]. However, the efficacy and safety of eradication therapy for healing ESDinduced gastric ulcers are not clear.

It is well known that $H$. pylori infection is one of the etiologies inducing gastric ulcers. In previous reports, the healing effect of $H$. pylori-eradication monotherapy on gastric ulcers in $H$. pylori-infected Japanese patients was not superior to 8-week PPI therapy [9]. Eradication monotherapy may be insufficient to heal ESD-induced gastric ulcers. Terano et al. investigated the efficacy of 7-week treatment with rebamipide, a gastro-protective anti-ulcer drug, in patients with gastric ulcers after $H$. pylori eradication therapy compared with placebo. Rebamipide promoted gastric ulcer healing regardless of the success or failure of $H$. pylori eradication [10]. Fujiwara et al. reported that combination therapy with PPI and rebamipide showed a superior healing effect compared with PPI in patients with ESD-induced gastric ulcers [11].

In a randomized control study, we compared a group treated with PPI for 8 weeks after ESD (group A; standard therapy) and a group treated with 1 week of eradication therapy after ESD and subsequently treated with rebamipide for 7 weeks (group B) in EGC patients who were positive for $H$. pylori.

\section{Methods}

Study setting

This multicenter, randomized, open-label study was conducted in Japan. This study was conducted in accordance with the ethical principles of the Declaration of Helsinki. The protocol was approved by the ethics committees at each study site. All patients provided written informed consent. This study was registered with UMIN: registration no. UMIN000003181.

\section{Patients}

Patients included in this study underwent ESD based on whether their $H$. pylori-infected EGC fit the criteria proposed by guidelines of the Japanese Gastric Cancer Association [12]. Patients who received oral nonsteroidal antiinflammatory drugs, continuous corticosteroids, or an antithrombotic drug, who had a complicated peptic ulcer, a history of GI surgery, or received $H$. pylori eradication therapy were excluded.

\section{Treatment}

Patients were randomized to two study groups (group A and B). All patients received intravenous administration of $40 \mathrm{mg}$ omeprazole on the first 2 days after ESD; then, the study drugs were administered. In group A, patients received $20 \mathrm{mg}$ of omeprazole daily for 56 days. In group B, patients received $20 \mathrm{mg}$ of omeprazole, $750 \mathrm{mg}$ of amoxicillin, and $400 \mathrm{mg}$ of clarithromycin twice daily for 7 days, and then $100 \mathrm{mg}$ of rebamipide three times daily for 49 days. Endoscopic examination was performed at day 2, 7, and 58 after ESD, and the artificial ulcer area was calculated. In addition, ulcer stages such as healing and scar stages were evaluated. In group B, the ${ }^{13} \mathrm{C}$-urea breath test (UBit ${ }^{\circledR}$; Otsuka, Tokyo, Japan) was performed on day 84 after ESD to confirm the presence or absence of $H$. pylori.

The presence of $H$. pylori infection was determined by histological evaluation (modified Giemsa staining), the rapid urease test (CLOtest ${ }^{\circledR}$; Kimberley-Clark, Draper, UT, USA), serum antibody test, or 13C-urea breath test. When one of these tests was positive, we would score the patient as positive for the presence of $H$. pylori infection. Eradication of $H$. pylori was defined as successful when the results of both the rapid urease test and histology were negative or when those of the 13C-urea breath test were negative, with the cutoff value of delta over baseline as $2.5 \%$. 
Sizes of the artificial ulcer were measured using the upper GI endoscopic measure (M2-4, Olympus, Tokyo, Japan). The ulcer area was presumed to be the approximate value of the ellipse; it is calculated by measuring the diameter of the longitude and transverse of the artificial ulcer. A scar was defined as the disappearance of the white coat in the center of the ulcer area. Bleeding was defined as hematemesis or melena that required endoscopic hemostasis and decreased the hemoglobin count by more than $2 \mathrm{~g} / \mathrm{dl}$, occurring from the time of treatment to within 56 days after ESD.

\section{Randomization}

A randomization code was assigned a treatment code corresponding to each study drug code by the allocation manager from the contract research organization for the registration center (Kondo Photo Process Co., Ltd., Osaka, Japan). The study drug allocation manager sealed the assignment list and kept it sealed until the designated time for unmasking.

\section{Evaluation}

The primary end point was to evaluate the healing ratio to scar stage to the final evaluation time point comparison between group A and B. Secondary end points were to evaluate the change in the reduction ratio of the artificial ulcer from baseline to the final evaluation time point comparison between the groups. The effect of the $H$. pylori eradication therapy (stratified according to success or failure) and artificial ulcer area at baseline (stratified according to $\geq$ median area or $<$ median area) was also evaluated. In addition, adverse events were also recorded. Baseline was defined as the measured value at day 2 after the ESD procedure, and the final evaluation time point was defined as the measured value at day 56. If the study drug treatment was not completed, the measured value at the time of dropout was defined as the final evaluation time point.

\section{Statistical analysis}

In a previous report, the healing ratio with rebamipide treatment at day 56 after $H$. pylori eradication therapy was $70 \%$ [10]. On the other hand, administration of PPI at 56 days after ESD was $100 \%$ [13]. Our hypothesis is that the difference in both groups is at least $10 \%$. Sample size was calculated based on this hypothesis. A two-sided test with a 0.05 significance level and $80 \%$ power $(\alpha=0.05$, $\beta=0.20$ ) would require 37 subjects per group.

The categorical data were analyzed by chi-square test, Fisher's exact test, or Mann-Whitney's $U$ test. The continuous data were analyzed by paired $t$ test.
Statistical significance was defined as $P<0.05$. All statistical analyses were performed using SAS Jump version 10.0.0 software (SAS Institute, Cary, NC).

\section{Results}

This study was conducted from April 2010 through March 2011 at nine study sites. Of 88 patients who received at least one dose of the study drug, 6 who deviated from the protocol and 2 at the physician's request were excluded from this study (Fig. 1). Eighty patients were randomized in this study. One patient in group A was excluded from all analysis sets because of loss to follow-up, and two patients in group B were excluded because of loss to follow-up. Forty patients in group A and 37 patients in group B completed treatment with the study drugs and were evaluated in all analysis sets. Patient's demographic data and characteristics did not show statistically significant differences between the groups (Table 1).

At the final evaluation time point, 34 of $40(85.0 \%)$ patients in group A and 21 of $37(56.8 \%)$ in group B $(P=0.0107)$ had progressed to the scar stage (Table 2).

The change in the reduction ratio of the artificial ulcer area at day 7 was $15.2 \pm 52.5 \%$ in group $A$ and $38.1 \pm 24.0 \%$ in group $\mathrm{B}$, respectively $(P=0.0195)$. The change in the reduction ratio of the artificial ulcer area at the final evaluation time point was $98.0 \pm 4.8 \%$ in group $\mathrm{A}$ and $92.3 \pm 12.0 \%$ in group $\mathrm{B}$, respectively $(P=0.0096)$ (Fig 2).

Change in progression to scar at the final evaluation time point by stratifying the ulcer size was evaluated. For those with a $>565.5 \mathrm{~mm}^{2}$ (median was $565.5 \mathrm{~mm}^{2}$ ) artificial ulcer area at baseline, 15 of $19(79.0 \%)$ patients were evaluated as being at the scar stage in group A and 7 of 18 $(59.5 \%)$ in group $\mathrm{B}$, respectively $(P=0.0131)$. In artificial ulcer areas of less than $565.5 \mathrm{~mm}^{2}$, there was no significant difference between the groups (Table 3).

The change in progressing to scar at the final evaluation time point stratifying for the effect of $H$. pylori eradication therapy was also evaluated. In case of $H$. pylori eradication, 34 of $40(85.0 \%)$ patients were evaluated as being at scar stage in group A and 17 of $29(58.6 \%)$ in group B, respectively $(P=0.0138)$. In case of failure, there was no statistical significance (Table 4).

\section{Adverse events}

Perforation was observed in one ESD patient in group A, and bleeding was observed in one patient at day 5 in group B. Diarrhea was observed in two patients in group B. 
Fig. 1 Patient flow chart

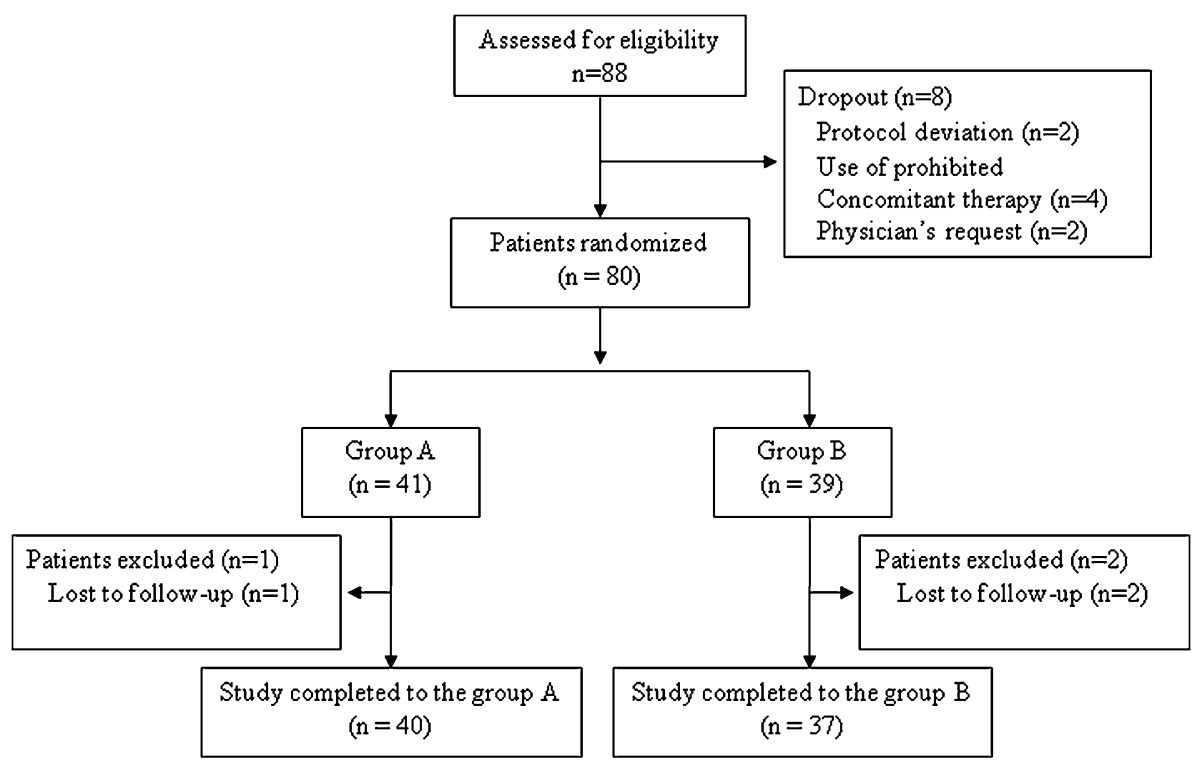

Table 1 Demographic and characteristic data

\begin{tabular}{|c|c|c|c|}
\hline & $\begin{array}{l}\text { Group A } \\
(n=41)\end{array}$ & $\begin{array}{l}\text { Group B } \\
(n=39)\end{array}$ & $P$ value \\
\hline \multicolumn{4}{|l|}{ Age } \\
\hline Mean $\pm \mathrm{SD}$ & $67 \pm 8$ & $66 \pm 7$ & $\mathrm{Ns}^{\mathrm{a}}$ \\
\hline \multicolumn{4}{|l|}{ Sex } \\
\hline Male & $29(70.7)$ & $28(71.8)$ & \multirow[t]{2}{*}{$\mathrm{Ns}^{\mathrm{b}}$} \\
\hline Female & $12(29.3)$ & $11(28.2)$ & \\
\hline \multicolumn{4}{|l|}{ Height } \\
\hline Mean $\pm \mathrm{SD}$ & $160.7 \pm 9.0$ & $163.2 \pm 9.2$ & $\mathrm{Ns}^{\mathrm{a}}$ \\
\hline \multicolumn{4}{|l|}{ Weight } \\
\hline Mean $\pm \mathrm{SD}$ & $59.8 \pm 9.4$ & $59.8 \pm 9.2$ & $\mathrm{Ns}^{\mathrm{a}}$ \\
\hline \multicolumn{4}{|l|}{ Smoking } \\
\hline No & $21(51.2)$ & $21(53.8)$ & \multirow[t]{2}{*}{$\mathrm{Ns}^{\mathrm{b}}$} \\
\hline Yes & $20(48.8)$ & $18(46.2)$ & \\
\hline \multicolumn{4}{|l|}{ Drinking } \\
\hline No & $15(36.6)$ & $14(35.9)$ & \multirow[t]{2}{*}{$\mathrm{Ns}^{\mathrm{b}}$} \\
\hline Yes & $26(63.4)$ & $25(64.1)$ & \\
\hline \multicolumn{4}{|l|}{ Medical history } \\
\hline No & $16(39.0)$ & $12(30.8)$ & \multirow[t]{2}{*}{$\mathrm{Ns}^{\mathrm{b}}$} \\
\hline Yes & $25(61.0)$ & $27(69.2)$ & \\
\hline \multicolumn{4}{|c|}{ Preexisting comorbidity } \\
\hline No & $32(78.0)$ & $30(76.9)$ & \multirow[t]{2}{*}{$\mathrm{Ns}^{\mathrm{b}}$} \\
\hline Yes & $9(22.0)$ & $9(23.1)$ & \\
\hline \multicolumn{4}{|c|}{ Other medication } \\
\hline No & $19(46.3)$ & $15(38.5)$ & \multirow[t]{2}{*}{$\mathrm{Ns}^{\mathrm{b}}$} \\
\hline Yes & $22(53.7)$ & $24(61.5)$ & \\
\hline
\end{tabular}

Data are expressed as mean \pm SD or number of patients (\%)

$S D$ standard deviation, $N S$ not significant

a Mann-Whitney $U$ test, ${ }^{\mathrm{b}}$ chi-square test
Table 2 Changes in gastric ulcer scar stage at 56 days after ESD

\begin{tabular}{lll}
\hline & Number of patients $(\%)$ & $P$ value \\
\hline Group A $(n=40)$ & $34(85.0)$ & 0.0107 \\
Group B $(n=37)$ & $21(56.8)$ & \\
\hline
\end{tabular}

Comparisons between group A and B were performed using Fisher's exact test

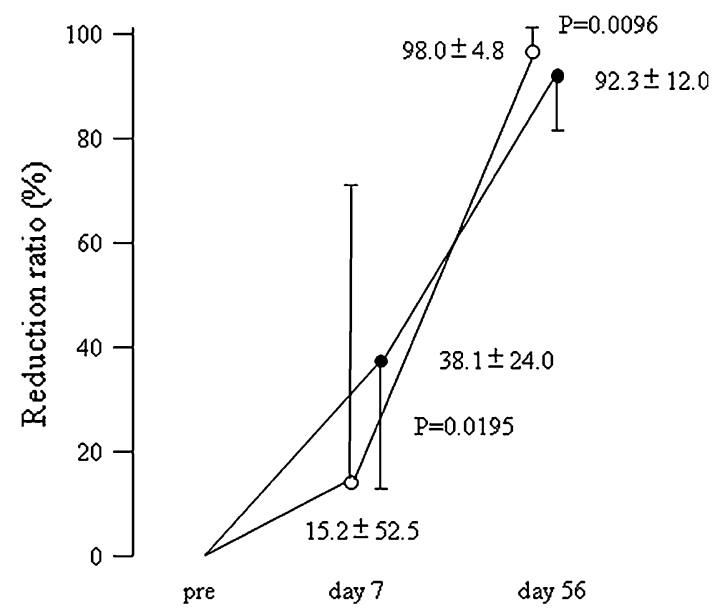

Fig. 2 Changes in the artificial ulcer area reduction ratio from baseline to day 56. The open circle indicates group A and the closed circle group B

\section{Discussion}

In the present study, we showed two obvious results. First, serious adverse events such as bleeding from the artificial ulcer after ESD were not observed in patients with $H$. 
Table 3 Change in gastric ulcer stage to scar at 56 days after ESD stratifying by ulcer size

\begin{tabular}{lcc}
\hline & Number of patients $(\%)$ & $P$ value \\
\hline$\geq 565.5 \mathrm{~mm}^{2}$ & & \\
Group A $(n=19)$ & $15(79.0)$ & 0.0131 \\
Group B $(n=18)$ & $7(59.5)$ & \\
$<565.5 \mathrm{~mm}^{2}$ & & 0.1628 \\
Group A $(n=21)$ & $19(90.5)$ & \\
Group B $(n=19)$ & $14(73.7)$ & \\
\hline
\end{tabular}

Comparisons between groups $\mathrm{A}$ and $\mathrm{B}$ were performed by using Fisher's exact test

Table 4 Change in gastric ulcer stage to scar at 56 days after ESD by stratifying the effect of $H$. pylori eradication therapy

\begin{tabular}{|c|c|c|}
\hline & Number of patients (\%) & $P$ value \\
\hline \multicolumn{3}{|c|}{ H. pylori eradication success } \\
\hline Group A $(n=40)$ & $34(85.0)$ & 0.0138 \\
\hline Group B $(n=29)$ & $17(58.6)$ & \\
\hline \multicolumn{3}{|c|}{ H. pylori eradication failure } \\
\hline Group A $(n=40)$ & $34(85.0)$ & 0.2692 \\
\hline Group B $(n=6)$ & $4(66.7)$ & \\
\hline
\end{tabular}

Comparisons between group A and B were carried out using Fisher's exact test

pylori eradication therapy compared with those with 8 weeks of PPI therapy as standard therapy for the artificial ulcer. No previous evidence had been reported about performing $H$. pylori eradication therapy after hemostatic treatment in active gastric ulcer patients with bleeding. In this study, performing $H$. pylori eradication therapy 3 days after ESD was safe without worsening the ulcers and without bleeding. Our result may suggest optimal timing of H. pylori eradication therapy as one of the options for patients who have undergone ESD.

Second, we showed that $H$. pylori eradication therapy plus 7 weeks of rebamipide monotherapy was not superior to PPI monotherapy. Since artificial ulcers were reduced by approximately $90 \%$ in both groups, a slightly longer term observation might be required for unhealed ulcers with small white coats at 8 weeks.

In artificial ulcer areas of less than $565.5 \mathrm{~mm}^{2}$, there was no significant difference between PPI monotherapy and $H$. pylori eradication therapy plus 7 weeks of rebamipide monotherapy. Therefore, rebamipide monotherapy following $H$. pylori eradication therapy was considered an optimal therapeutic option in patients with smaller sized artificial ulcers $\left(<565.5 \mathrm{~mm}^{2}\right)$. In patients with artificial ulcer areas of more than $565.5 \mathrm{~mm}^{2}, 7$ weeks of PPI therapy in place of rebamipide might be considered an optimal option following $H$. pylori eradication therapy. Also, we investigated the healing effect of combination therapy including rabeprazole or teprenone with $H$. pylori eradication in ESD patients with artificial ulcers [14]. The teprenone group did not have better results than the rabeprazole group in patients with ulcers of more than $1.5 \mathrm{~cm}$ diameter. Thus, limitations may exist to the use of combination therapy (especially H. pylori eradication plus cytoprotective agents) in patients with large artificial ulcers after ESD.

There was only one report on the effect of Helicobacter pylori eradication monotherapy on gastric ulcer healing after endoscopic mucosal resection. Cheon et al. found that the ulcer reduction ratio was significantly higher in the $H$. pylori eradication group compared with the PPI group at 4 weeks after endoscopic mucosal resection [8]. Moreover, no serious adverse events such as bleeding were observed. The end point of their study was the ulcer reduction, not healing, ratio at 4 weeks after EMR, not ESD. In the present study, the reduction ratio of the artificial ulcer area at day 7 in the eradication plus rebamipide group was higher than that in the PPI group. Since eradication therapy is treated with double-dose PPI, the ulcer reduction ratio in the initial phase (from 1 to 4 weeks) may be higher than that in the PPI group. Concerning the term of administration, Niimi et al. [15] reported that 2-week administration of PPI for post-ESD gastric ulcers may be sufficient to aid healing without increasing adverse effects. This report may suggest obtaining sufficient efficacy by adding 2-week administration of PPI in combination therapy including eradication therapy and a cytoprotective agent. Eradication can be performed at many different times (e.g., before or just after ESD, after ulcer healing), but the pros and cons of treatment timing have not been investigated in a formal study. Our study is significant in that it provides some evidence to consider when debating the pros and cons of eradication therapy just after ESD. Given that a double dose of PPI during eradication therapy may promote ulcer healing, using rebamipide after eradication, unlike PPIs, allows assessment of eradication 8 weeks after ESD (when ulcer healing is checked). This reduces costs and patient burden. Unless eradication just after ESD is shown to have drawbacks, this treatment method may be a viable option. A limitation of this study was not evaluating the comparison of $H$. pylori eradication monotherapy and PPI therapy. We previously demonstrated the efficacy of $H$. pylori eradication therapy compared with PPI therapy for gastric ulcers. H. pylori eradication therapy was weaker than PPI monotherapy. Furthermore, in the $H$. pylori eradication therapy group, adverse events such as bleeding have been observed in some patients [9]. Because of ethical considerations, we conducted the present study to investigate the effect of rebamipide following $H$. pylori eradication 
therapy compared with PPI monotherapy. In this study, the sample size was small. However, we confirmed H. pylori eradication therapy plus 7 weeks rebamipide monotherapy was not superior to PPI monotherapy. In the next step, a comparative study of 8-week PPI therapy and 7-week PPI therapy following $H$. pylori eradication therapy is needed.

In conclusion, $H$. pylori eradication therapy plus 7 -week rebamipide monotherapy was not superior to PPI monotherapy, but eradication plus rebamipide therapy for smaller sized artificial ulcers was an optimal therapeutic option for healing. Serious adverse events were not observed in eradication plus rebamipide therapy compared with PPI therapy.

Conflict of interest Yuji Naito received scholarship funds from Otsuka Pharmaceutical Co., Ltd., Eisai Co., Ltd., and Takeda Pharmaceutical Co., Ltd., for basic research that was not related to the present clinical study. Nobuaki Yagi has an affiliation with a donation-funded department of AstraZeneca Co., Ltd., Eisai Co., Ltd., Otsuka Pharmaceutical Co., Ltd., MSD K.K., Dainippon Sumitomo Pharma Co., Ltd., Chugai Pharmaceutical Co., Ltd., FUJIFILM Medical Co., Ltd., and Merck Serono Co., Ltd. Takeshi Azuma has an affiliation with a donation-funded department from Otsuka Pharmaceutical Co., Ltd., and MSD K.K.; Takeshi Azuma serves as a consultant to Eisai Co., Ltd.

\section{References}

1. Tada M, Murakami A, Karita M, Yanai H, Okita K. Endoscopic resection of early gastric cancer. Endoscopy. 1993;25:445-50.

2. Inoue H, Takeshita K, Hori H, Muraoka Y, Yoneshima H, Endo M. Endoscopic mucosal resection with a cap-fitted panendoscope for esophagus, stomach, and colon mucosal lesions. Gastrointest Endosc. 1993;39:58-62.

3. Ono $\mathrm{H}$, Kondo $\mathrm{H}$, Gotoda $\mathrm{T}$, et al. Endoscopic mucosal resection for treatment of early gastric cancer. Gut. 2001;48:225-9.

4. Uemura N, Okamoto S, Yamamoto S, et al. Helicobacter pylori infection and the development of gastric cancer. N Engl J Med. 2001;345:784-9.

5. Fukase K, Kato M, Kikuchi S, et al. Effect of eradication of Helicobacter pylori on incidence of metachronous gastric carcinoma after endoscopic resection of early gastric cancer: an open-label, randomized controlled trial. Lancet. 2008;372:392-7.

6. Yamaguchi Y, Katsumi N, Tauchi M, et al. A prospective randomized trial of either famotidine or omeprazole for the prevention of bleeding after endoscopic mucosal resection and the healing of endoscopic mucosal resection-induced ulceration. Aliment Pharmacol Ther. 2005;21(Suppl 2):111-5.

7. Kakushima N, Yahagi N, Fujishiro M, et al. The healing process of gastric artificial ulcers after endoscopic submucosal dissection. Dig Endosc. 2004;16:327-31.

8. Cheon Jae Hee, Kim Jie-Hyun, Lee Sang Kil, 11 Kim Tae, Kim Won Ho, Lee Yong Chan. Helicobacter pylori eradication therapy may facilitate gastric ulcer healing after endoscopic mucosal resection: a prospective randomized study. Helicobacter. 2008; 13(6):564-71.

9. Higuchi K, Fujiwara K, Tominaga K, Watanabe M, Shiba S, Nakamura $\mathrm{S}$, et al. Is eradication sufficient to heal gastric ulcer in patients infected with Helicobacter pylori? A randomized, controlled, prospective study. Aliment Pharmacol Ther. 2003;17:111-7.

10. Terano A, Arakawa T, Sugiyama T, Suzuki H, Joh T, Yoshikawa T, Higuchi K, Haruma K, Murakami K, Kobayashi K. Rebamipide Clinical Study Group. Rebamipide, a gastro-protective and anti-inflammatory drug, promotes gastric ulcer healing following eradication therapy for Helicobacter pylori in a Japanese population: a randomized, double-blind, placebo-controlled trial. J Gastroenterol. 2004;2(8):690-3.

11. Fujiwara S, Morita Y, Toyonaga T, Kawakami F, Itoh T, Yoshida M, Kutsumi H, Azuma T. A randomized controlled trial of rebamipide plus rabeprazole for the healing of artificial ulcers after endoscopic submucosal dissection. J Gastroenterol. 2011;46(5): 595-602.

12. Guidelines for diagnosis and treatment of carcinoma of stomach. Japanese Gastric Cancer Society 2004.

13. Takeuchi N, Umegaki E, Takeuchi T, Murano Yukiko Yoda M, Tokioka S, Higuchi K. Gastric ulcer healing after treatment of endoscopic submucosal dissection in Japanese: comparison of $\mathrm{H} 2$ receptor antagonist and proton pump inhibitor administration. J Clin Biochem Nutr. 2011;49(3):216-21.

14. Takeuchi T, Umegaki E, Takeuchi N, Yoda Y, Kojima Y, Tokioka S, Higuchi K. Strategies for peptic ulcer healing after 1 week proton pump inhibitor-based triple Helicobacter pylori eradication therapy in Japanese patients : differences of gastric ulcers and duodenal ulcers. J Clin Biochem Nutr. 2012;51(3): 189-95.

15. Niimi K, Fujishiro M, Goto O, et al. prospective single-arm trial of two-week rabeprazole treatment for ulcer healing after gastric endoscopic submucosal dissection. Dig Endosc. 2012;24(2): $110-6$. 